\title{
Preoperative high-dose atorvastatin for prevention of atrial fibrillation after cardiac surgery: A randomized controlled trial
}

\author{
Antonios Kourliouros, MRCS, ${ }^{\mathrm{a}}$ Oswaldo Valencia, MD, ${ }^{\mathrm{a}}$ Morteza Tavakkoli Hosseini, MD, ${ }^{\mathrm{a}}$ \\ Manuel Mayr, MD, PhD, ${ }^{\mathrm{b}}$ Mazin Sarsam, FRCS, ${ }^{\mathrm{a}}$ John Camm, FRCP, ${ }^{\mathrm{a}}$ and Marjan Jahangiri, FRCS ${ }^{\mathrm{a}}$
}

\begin{abstract}
Objective: The preventative effect of statins on postoperative atrial fibrillation has been hypothesized. However, all studies to date have examined patients who did not receive statins before their further allocation to treatment or no treatment. Because guidelines recommend the routine use of statins in patients with coronary artery disease, we set out to examine the effect of intensive statin pretreatment versus continuation of usual statin dose on atrial fibrillation after cardiac surgery.
\end{abstract}

\begin{abstract}
Methods: Patients receiving routine statin treatment and undergoing coronary artery bypass surgery or aortic valve replacement with no history of atrial fibrillation or antiarrhythmic medication were randomized to receive atorvastatin $80 \mathrm{mg}$ or atorvastatin $10 \mathrm{mg}$ for 7 days before surgery in a single-blind fashion. The primary end point was the development of postoperative atrial fibrillation during hospital stay.
\end{abstract}

Results: A total of 104 consecutive patients were included. Postoperative atrial fibrillation occurred in 33 patients $(32.4 \%)$. No significant differences were found in demographics, medical history, or intraoperative variables between treatment groups, with the exception of higher rate of $\beta$-blocker use in the atorvastatin $10 \mathrm{mg}$ group (75\% vs $53 \%, P=.002)$ and previous myocardial infarction ( $62 \%$ vs $42 \%, P=.049)$. The incidence of postoperative atrial fibrillation was lower in the atorvastatin $80 \mathrm{mg}$ group when compared with the atorvastatin $10 \mathrm{mg}$ group, but this difference did not reach statistical significance $(29 \%$ vs $36 \%, P=.43)$.

Conclusions: High-dose atorvastatin for 7 days before cardiac surgery conferred a nonsignificant reduction in postoperative atrial fibrillation when compared with a low-dose regimen. A larger study would be necessary to confirm the beneficial effect of high-dose statins in this setting. (J Thorac Cardiovasc Surg 2011;141:244-8)

Atrial fibrillation (AF) after cardiac surgery is associated with prolonged hospital stay, increased overall morbidity, and long-term mortality. ${ }^{1}$ The identification of agents with a potential modulatory effect on the substrate responsible for AF has led to clinical studies assessing the applicability of these "upstream therapies" in the prevention of postoperative $\mathrm{AF}^{2}$. The antiarrhythmic role of statins has previously been examined against the background of different cardiovascular conditions with variable results. The preventative effect of statins on the development of AF after cardiac surgery has also been suggested, based on various observational studies, randomized controlled trials, and meta-analyses. ${ }^{3}$ Our group previously validated that statins could be protective against postoperative $\mathrm{AF}$, but, more important, that this effect is dose-dependent. ${ }^{4}$

In all prospective controlled studies to date, the potential prophylactic effect of statins was examined against placebo

From the Department of Cardiothoracic Surgery, ${ }^{\text {a }}$ St George's Hospital, London, United Kingdom; and Cardiovascular Division, ${ }^{\mathrm{b}}$ King's College London, United Kingdom.

Disclosures: Authors have nothing to disclose with regard to commercial support. Received for publication Jan 21, 2010; revisions received April 7, 2010; accepted for publication June 7, 2010; available ahead of print July 12, 2010.

Address for reprints: Marjan Jahangiri, FRCS, Department of Cardiothoracic Surgery, St George's Hospital, Blackshaw Road, London SW17 0QT, United Kingdom (E-mail: marjan.jahangiri@stgeorges.nhs.uk).

0022-5223/\$36.00

Copyright (c) 2011 by The American Association for Thoracic Surgery doi:10.1016/j.jtcvs.2010.06.006 or nontreatment control groups. ${ }^{5,6}$ Although this observation is of great research interest, its clinical applicability is limited because the majority of patients undergoing cardiac surgery receive statins as part of their disease management. Therefore, and in view of the possible doserelated effect of statins, we set out to examine the impact of 7-day preoperative treatment with high $(80 \mathrm{mg})$ versus usual $(10 \mathrm{mg})$ doses of atorvastatin in the development of postoperative AF in patients who had previously received routine statin therapy. Thus, the prospective role of intensive statin treatment in the prevention of AF after cardiac surgery was studied, with the potential to influence routine clinical practice in the perioperative management of these patients.

\section{MATERIALS AND METHODS}

This study was a randomized, controlled, single-blinded trial examining the effect of 7-day pretreatment with atorvastatin $80 \mathrm{mg}$ versus atorvastatin $10 \mathrm{mg}$ on the incidence of AF after cardiac surgery in patients already receiving routine statin treatment. The primary end point was the development of postoperative AF during hospital stay. Ethical approval was obtained, and all participants gave informed written consent. Approval from Medicines and Healthcare products Regulatory Agency was also obtained (EudraCT 2006-005272-40). This study was included in the International Standard Randomised Controlled Trial Number Register (41309956). The design, analysis, and reporting of data derived from this randomized controlled trial were carried out according to CONSORT recommendations. ${ }^{7}$

A total of 123 consecutive patients undergoing elective first-time coronary artery bypass grafting (CABG) or aortic valve replacement by a single surgeon were randomized in a single-blind fashion between April 2007 and 


\section{Abbreviations and Acronyms \\ $\mathrm{AF} \quad=$ atrial fibrillation \\ $\mathrm{CABG}=$ coronary artery bypass grafting}

April 2009. Patients already receiving statins at the usual treatment dose (atorvastatin 10 or $20 \mathrm{mg}$; simvastatin 10,20 , or $40 \mathrm{mg}$ ) were randomized to atorvastatin 10 or $80 \mathrm{mg}$. Patients receiving intensive statin therapy (atorvastatin $40 \mathrm{or} 80 \mathrm{mg}$; simvastatin $80 \mathrm{mg}$ ) before surgery were excluded. Additional exclusion criteria were as follows: history of atrial arrhythmias; treatment with antiarrhythmic agents (except $\beta$-blockers); valve surgery other than aortic; hepatobiliary disease; unexplained persistent elevations in serum transaminases; increased consumption of alcohol; consistent drinking more than $4 \mathrm{U} / \mathrm{d}$; serum creatinine greater than $180 \mathrm{mmol} / \mathrm{L}$; known intolerance to statins or myopathy; cyclosporin, fibric acid derivatives, erythromycin, niacin, or azole antifungal use; and pregnant or breastfeeding women.

Patients were evaluated in a preassessment clinic at St George's Hospital, London, at least 2 weeks before surgery and were randomized at the time. Statins were delivered to the patient in an appropriately numbered container by a member of administrative staff, independent of the trial. Allocated atorvastatin treatment was then commenced 7 days before surgery, and the usual statin therapy was discontinued at that time. Study medication was continued for 14 days after surgery or until discharge (whichever first). Daily administration of statins during hospital stay was carried out by the on-duty nursing staff. Patients were reassessed on completion of the trial medication just before hospital discharge or at 2 weeks after surgery and were prescribed their preoperative statin type and dose. At this point, the same (independent of the trial) member of administrative staff received the empty skillet and blisters from the patient and checked compliance. Thus, ensuring that the investigator was not inadvertently unblinded during the course of the study (skillets were never opened and tablets were administered in the presence of an investigator). In addition, a tamper-evident seal on the external skillet was applied to secure blinding.

Randomization was performed by an international clinical trials agency (Richmond Pharmacology, London, UK) that provided the statistician, randomization algorithm (computerized random number generation), and external quality assurance.

Standardized surgical and anesthetic protocols were used as published previously. ${ }^{8}$ Routine postoperative heart rhythm assessment was achieved with continuous telemonitoring for the first 72 hours after surgery and with 4-hourly clinical examinations thereafter. Twelve-lead electrocardiograms were also obtained and assessed daily.

\section{Statistical Analysis and Sample Size Calculation}

To estimate the appropriate sample size for difference in proportions (development of postoperative $\mathrm{AF}$ ) in equally sized treatment groups, data were obtained from an observational study previously carried out by our group. ${ }^{4}$ Having examined the variable effect of different statin doses, we hypothesized that the high-dose treatment group would exhibit an incidence of $\mathrm{AF}$ in the same region, at least, as the one previously observed with simvastatin $40 \mathrm{mg}(15.6 \%)$. Conversely, we anticipated that the incidence of AF in the low-dose group (atorvastatin $10 \mathrm{mg}$ ) would be similar to the one achieved by the same type and dose in the observational study $(41.9 \%)$. On the basis of an estimated proportion for postoperative AF of $16 \%$ $(p 1)$ in the atorvastatin $80 \mathrm{mg}$ group and a proportion of $42 \%(p 2)$ in the atorvastatin $10 \mathrm{mg}$ group, sample size was calculated using the following established formula. ${ }^{9}$ Note that $n$ equals the number of subjects required in each group and that the coefficient $C_{p}$, power equals 10.5 to achieve a $P$ value of .05 and an alpha power of $90 \%$.
TABLE 1. Patient characteristics and operative variables between the low- and high-dose statin groups

\begin{tabular}{lccc}
\hline \multicolumn{1}{c}{ Variable } & $\begin{array}{c}\text { Atorvastatin } \\
\mathbf{1 0} \mathbf{~} \mathbf{g}(\mathbf{n}=\mathbf{5 3})\end{array}$ & $\begin{array}{c}\text { Atorvastatin } \\
\mathbf{8 0 ~} \mathbf{~ m g}(\mathbf{n}=\mathbf{4 9})\end{array}$ & $\begin{array}{c}\boldsymbol{P} \\
\text { value }\end{array}$ \\
\hline Age (y) & $66 \pm 9.2$ & $66 \pm 8.5$ & .95 \\
Male & $47(89)$ & $42(86)$ & .65 \\
Medical history & & & \\
$\quad$ Hypertension & $36(68)$ & $33(67)$ & .95 \\
Diabetes & $12(23)$ & $8(16)$ & .42 \\
Previous TIA & $1(2)$ & $4(8)$ & .19 \\
COPD & $4(8)$ & $4(8)$ & 1.00 \\
Medications & & & \\
$\beta$-blockers & $40(75)$ & $26(53)$ & .018 \\
ACEIs & $38(72)$ & $29(59)$ & .18 \\
Cardiac history & & & \\
CCS class III-IV & $5(9)$ & $8(16)$ & .55 \\
NYHA class III-IV & $8(15)$ & $79(16)$ & .47 \\
Previous MI & $33(62)$ & $21(43)$ & .05 \\
LVEF $<50 \%$ & $13(25)$ & $10(20)$ & .62 \\
Operative characteristics & & & \\
CABG & $47(89)$ & $46(94)$ & .49 \\
Off-pump & $13(25)$ & $13(27)$ & .82 \\
CABG+AVR & $3(6)$ & $1(2)$ & .62 \\
AVR & $9(17)$ & $4(8)$ & .18 \\
CPB time (min) & $65 \pm 44$ & $65 \pm 51$ & .84 \\
Crossclamp time (min) & $41 \pm 28$ & $41 \pm 31$ & .81 \\
euroSCORE & $2.87 \pm 1.7$ & $2.63 \pm 1.7$ & .56 \\
Postoperative AF & $19(35.9)$ & $14(28.6)$ & .43 \\
\hline
\end{tabular}

TIA, Transient ischemic attack; COPD, chronic obstructive pulmonary disease; $A C E I$ angiotensin-converting enzyme inhibitor; CCS, Canadian Cardiovascular Society; $N Y H A$, New York Heart Association; $M I$, myocardial infarction; $L V E F$, left ventricular ejection fraction; $C A B G$, coronary artery bypass graft; $A V R$, aortic valve replacement; $C P B$, cardiopulmonary bypass; euroSCORE, European System for Cardiac Operative Risk Evaluation; $A F$, atrial fibrillation. Categoric data are numbers (\%); continuous data as means \pm standard deviation

$$
n=\frac{\left[p_{1}\left(1-p_{1}\right)+p_{2}\left(1-p_{2}\right)\right]}{\left(p_{1}-p_{2}\right)} \times \mathrm{C}_{p, p o w e r}
$$

For the values $p_{1}=0.16, p_{2}=0.42$ and $\mathrm{C}_{p, \text { power }}=10.5$ we calculate $n=58.7$

A total population of 123 patients was decided on the basis of a 1:1 randomization, allowing for the subsequent exclusion of 5 patients in case of refusal to participate after allocation, cancellations, and so forth.

Univariate analysis was performed, with postoperative AF being the dependent variable according to high or low atorvastatin dose. Dichotomous data were evaluated using Pearson's chi-square test or Fisher's exact test, whereas the $t$ test and Mann-Whitney $U$ test were used for continuous variables with normal or nonparametric distribution, respectively. Variables with statistical significance less than 0.2 (with the inclusion of atorvastatin $80 \mathrm{mg}$ use) were entered into a multivariate regression model to identify independent predictors of postoperative AF. Statistical analysis was performed with SPSS 17.0 software (SPSS Inc, Chicago, Ill).

\section{RESULTS}

A total of 102 patients were analyzed, 53 of whom received atorvastatin $10 \mathrm{mg}$ and 49 of whom received atorvastatin $80 \mathrm{mg}$. Patient characteristics and operative variables between the 2 treatment groups are shown in Table 1 . Twenty-one patients were excluded from the analysis. 


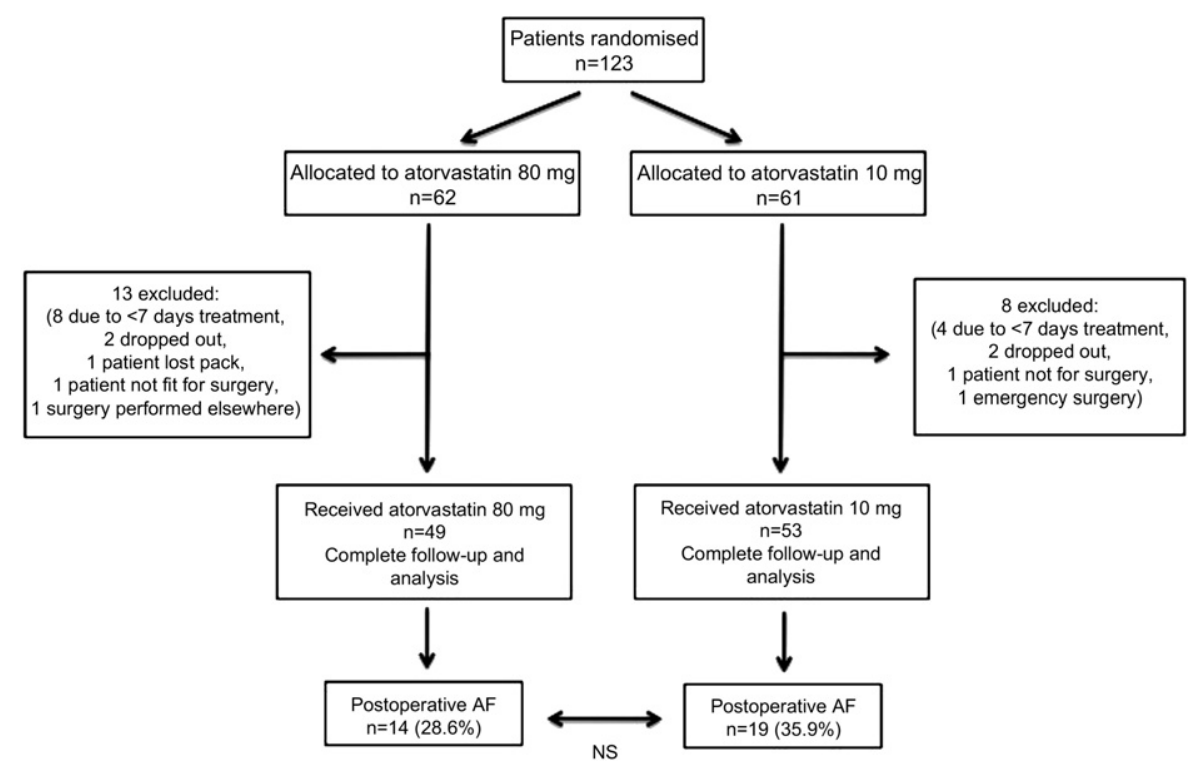

FIGURE 1. Flow diagram of randomized controlled trial. $A F$, Atrial fibrillation.

In the majority of cases $(n=12)$, patients did not complete the full 7 days of atorvastatin treatment before surgery, mainly because of unforeseen changes in the operating schedule. For the remaining 9 patients, 4 decided not to take the trial medication and continue with their standard statin, 1 lost the pack containing the atorvastatin, 1 underwent emergency surgery, 1 underwent surgery in a different hospital, and 2 decided not to undergo surgery. A flow diagram of the randomized controlled trial is shown in Figure 1.

No significant differences were found in demographics, medical history, or intraoperative variables between treatment groups, with the exception of higher rate of $\beta$-blocker use in the atorvastatin $10 \mathrm{mg}$ group $(75 \%$ vs $53 \%, P=.002)$ and previous myocardial infarction $(62 \%$ vs $42 \%$, $P=.049)$. The incidence of postoperative AF was lower in the atorvastatin $80 \mathrm{mg}$ group when compared with atorvastatin $10 \mathrm{mg}$, but this difference was not statistically significant $(29 \%$ vs $36 \%, P=.43)$ (Figure 2).

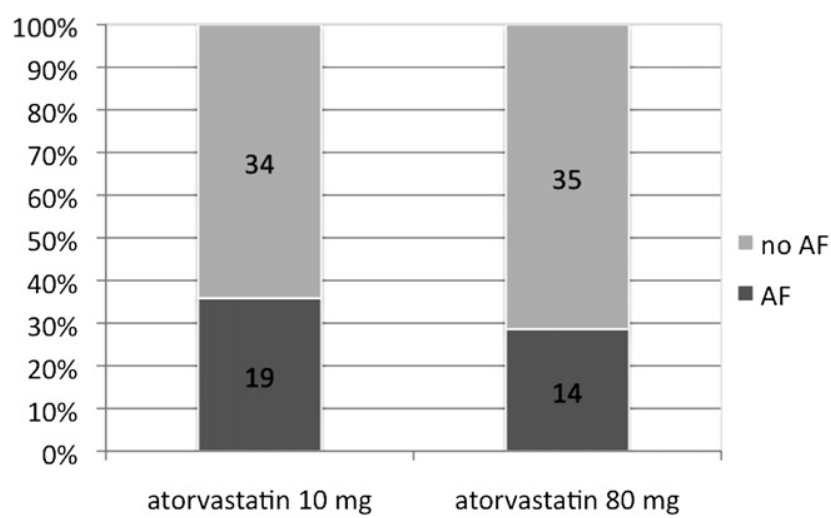

FIGURE 2. Incidence of postoperative AF according to atorvastatin dose. $A F$, Atrial fibrillation.
Patient characteristics with the inclusion of atorvastatin 80 $\mathrm{mg}$ as an independent variable were entered into a multivariate regression model to identify independent predictors of AF development. Use of high-dose atorvastatin was rejected at step 7 (of 13 steps) of the logistic regression. The remaining independent variables associated with postoperative $\mathrm{AF}$ are shown in Table 2.

The following postoperative complications occurred while patients were still receiving atorvastatin treatment and were promptly reported to the appropriate authorities: One patient died of myocardial infarction 3 days after CABG (received atorvastatin $10 \mathrm{mg}$, and AF developed on the first postoperative day), and 1 patient had a stroke during the hospital stay (received atorvastatin $80 \mathrm{mg}$ ).

\section{DISCUSSION}

Evidence suggests a beneficial effect of statins on the prevention of postoperative $\mathrm{AF}$ based on observational studies, ${ }^{4,10-14} 2$ randomized controlled trials, ${ }^{5,6}$ and 2 metaanalyses. ${ }^{15,16}$ Because of the consistency of the results from these studies, statin use is a recommended modality for the prevention of AF after cardiac surgery. ${ }^{2}$ More recently, however, a retrospective propensity-adjusted analysis by Virani and colleagues ${ }^{17}$ showed no effect of statin use on postoperative AF, whereas in a similar study by Miceli and colleagues, ${ }^{18}$ statins were found to be positively associated with AF after CABG. The differential risk profile of patients receiving statins during this 10 -year period and the known underreporting of arrhythmia outcomes in various registries may have led to this controversial result.

A common denominator of all relevant studies to date is that the possible effect of statins on postoperative AF was examined against patients who did not receive any statins 
TABLE 2. Independent predictors of postoperative atrial fibrillation

\begin{tabular}{|c|c|c|c|c|c|}
\hline Variable & Coefficient & Standard error & Odds ratio & $95 \%$ CI & $P$ value \\
\hline CCS score & 0.70 & 0.36 & 2.02 & $1.00-4.07$ & .049 \\
\hline Crossclamp time & 0.05 & 0.02 & 1.05 & $1.00-1.09$ & .048 \\
\hline CABG & -2.79 & 1.30 & 0.06 & $0.01-0.70$ & .032 \\
\hline Age & 0.12 & 0.04 & 1.13 & $1.05-1.21$ & .002 \\
\hline Constant & -11.13 & 3.30 & - & - & $<.001$ \\
\hline
\end{tabular}

$C I$, Confidence interval; $C C S$, Canadian Cardiovascular Society; $C A B G$, coronary artery bypass grafting.

preoperatively. It is recommended that statins be used in all patients with coronary artery disease undergoing surgery, irrespective of their lipid profile, unless contraindicated. ${ }^{19}$ As a result, even in earlier retrospective studies the control nostatin group comprised less than $20 \%$ of the overall cohort. This is why the identification of the beneficial effect of statins on postoperative $\mathrm{AF}$ over placebo or no treatment has limited applicability in day-to-day practice.

This study sought to investigate whether intensive atorvastatin pretreatment could confer significant reduction in postoperative $\mathrm{AF}$ over continuation of usual atorvastatin dose in patients who already received routine statin therapy. In addition to validating the potential antiarrhythmic role of statins in this setting, this study aimed at the development of an efficacious modality to complement the otherwise limited armamentarium against postoperative $\mathrm{AF}$.

The identified $7.3 \%$ absolute risk reduction in the odds of postoperative AF conferred by atorvastatin $80 \mathrm{mg}$ is in line with our original hypothesis. However, the lack of statistical significance $(P=.43)$ limits any definitive conclusions from being drawn. Despite the use of unrestricted randomization code and blinding of the investigator to participant treatment allocation, a higher rate of $\beta$-blocker use was found in the atorvastatin $10 \mathrm{mg}$ group compared with the atorvastatin 80 group (75\% vs $53 \%, P=.018)$. Previous studies have postulated the protective role of preoperative $\beta$-blockade in postoperative $\mathrm{AF}^{20}$ and this can partially explain the smaller treatment effect of high-dose statins than that suggested in our original hypothesis, which also determined the sample size. For such an observed difference between the 2 treatment groups, an adequately powered study would have required more than 650 patients to achieve a $P$ value of .05 with an $\alpha$ power of 0.80 . However, we can speculate that if baseline $\beta$-blocker use was similar or standardized, then patients receiving low-dose atorvastatin would exhibit higher rates of postoperative AF (as shown in the observational study) and the treatment effect of intensive dose atorvastatin would possibly be more pronounced.

\section{Study Limitations}

The decision for the number of subjects per treatment group was made on appropriate power size calculation with the allowance for approximately $5 \%$ of cancellation and dropout rates. This rate was exceeded because 21 pa- tients were excluded after randomization, mainly because of unanticipated changes in the operating schedule leading to fewer days of treatment with the investigational medication. Inclusion of these patients, however, would not have led to significantly different outcomes based on the treatment effect observed by high-dose statins. Although all measures were taken for the investigators not to be inadvertently unblinded, we accept that a double-blind study may have eliminated any detection bias in the trial. In line with most relevant studies to date, the method used for postoperative AF monitoring may have led to underdetection of some asymptomatic, self-terminating episodes of AF after the first 72 hours where continuous telemonitoring was used.

\section{CONCLUSIONS}

Although baseline lipid profile was routinely assessed before further allocation to investigational statin, data from follow-up measurements were incomplete and therefore not included in the analysis. The exact mechanism by which statins may protect against AF was beyond the scope of this study. However, we concur with previous investigators who have suggested that the potential antiarrhythmic effect of statins is independent of their lipid-lowering capacity. ${ }^{21}$ In addition, we previously showed that equivalent statin doses and types, based merely on their lipid-lowering efficacy, exhibit a differential effect on AF prevention. ${ }^{22}$ Consequently, we could not fully control for the impact of previously received routine treatment with statins from that achieved by the investigational dose atorvastatin.

\section{References}

1. Mariscalco G, Klersy C, Zanobini M, Banach M, Ferrarese S, Borsani P, et al. Atrial fibrillation after isolated coronary surgery affects late survival. Circulation. 2008;118:1612-8.

2. Kourliouros A, Savelieva I, Jahangiri M, Camm AJ. Atrial fibrillation: upstream therapies. In: Yusuf S, Cairns J, Camm AJ, Fallen EL, Gersh BJ, eds. EvidenceBased Cardiology. 3rd ed. London: BMJ Books; 2009:568-80.

3. Savelieva I, Kourliouros A, Camm J. Primary and secondary prevention of atrial fibrillation with statins and polyunsaturated fatty acids: review of evidence and clinical relevance. Naunyn Schmiedebergs Arch Pharmacol. 2009 [Epub ahead of print].

4. Kourliouros A, De Souza A, Roberts N, Marciniak A, Tsiouris A, Valencia O, et al. Dose-related effect of statins on atrial fibrillation after cardiac surgery. Ann Thorac Surg. 2008;85:1515-20.

5. Patti G, Chello M, Candura D, Pasceri V, D'Ambrosio A, Covino E, et al. Randomized trial of atorvastatin for reduction of postoperative atrial fibrillation in patients undergoing cardiac surgery: results of the ARMYDA-3 (Atorvastatin for Reduction of MYocardial Dysrhythmia After cardiac surgery) study. Circulation. 2006;114:1455-61. 
6. Song YB, On YK, Kim JH, Shin DH, Kim JS, Sung J, et al. The effects of atorvastatin on the occurrence of postoperative atrial fibrillation after off-pump coronary artery bypass grafting surgery. Am Heart J. 2008;156:373.e9-16.

7. Moher D, Schulz KF, Altman DG. The CONSORT statement: revised recommendations for improving the quality of reports of parallel-group randomised trials. Lancet. 2001;357:1191-4.

8. Kourliouros A, Valencia O, Phillips SD, Collinson PO, van Besouw JP, Jahangiri M. Low cardiopulmonary bypass perfusion temperatures are associated with acute kidney injury following coronary artery bypass surgery. Eur J Cardiothorac Surg. 2009 [Epub ahead of print].

9. Altman DG. Chapter 15. In: Altman D, ed. Practical Statistics for Medical Research. London: Chapman \& Hall; 1991:455.

10. Dotani MI, Elnicki DM, Jain AC, Gibson CM. Effect of preoperative statin therapy and cardiac outcomes after coronary artery bypass grafting. Am J Cardiol. 2000;86:1128-30.

11. Marin F, Pascual DA, Roldan V, Arribas JM, Ahumada M, Tornel PL, et al. Statins and postoperative risk of atrial fibrillation following coronary artery bypass grafting. Am J Cardiol. 2006;97:55-60.

12. Ozaydin M, Dogan A, Varol E, Kapan S, Tuzun N, Peker O, et al. Statin use before by-pass surgery decreases the incidence and shortens the duration of postoperative atrial fibrillation. Cardiology. 2007;107:117-21.

13. Mariscalco G, Lorusso R, Klersy C, Ferrarese S, Tozzi M, Vanoli D, et al. Observational study on the beneficial effect of preoperative statins in reducing atrial fibrillation after coronary surgery. Ann Thorac Surg. 2007;84:1158-64.

14. Lertsburapa K, White CM, Kluger J, Faheem O, Hammond J, Coleman CI Preoperative statins for the prevention of atrial fibrillation after cardiothoracic surgery. J Thorac Cardiovasc Surg. 2008;135:405-11.
15. Liakopoulos OJ, Choi YH, Haldenwang PL, Strauch J, Wittwer T, Dorge H, et al. Impact of preoperative statin therapy on adverse postoperative outcomes in patients undergoing cardiac surgery: a meta-analysis of over 30,000 patients. Eur Heart J. 2008;29:1548-59.

16. Liakopoulos OJ, Choi YH, Kuhn EW, Wittwer T, Borys M, Madershahian N, et al. Statins for prevention of atrial fibrillation after cardiac surgery: a systematic literature review. J Thorac Cardiovasc Surg. 2009;138:678-86.

17. Virani SS, Nambi V, Razavi M, Lee VV, Elayda M, Wilson JM, et al. Preoperative statin therapy is not associated with a decrease in the incidence of postoperative atrial fibrillation in patients undergoing cardiac surgery. Am Heart J. 2008;155: 541-6.

18. Miceli A, Fino C, Fiorani B, Yeatman M, Narayan P, Angelini GD, et al. Effects of preoperative statin treatment on the incidence of postoperative atrial fibrillation in patients undergoing coronary artery bypass grafting. Ann Thorac Surg. 2009;87: 1853-8.

19. Grundy SM, Cleeman JI, Merz CN, Brewer HB Jr, Clark LT, Hunninghake DB, et al. Implications of recent clinical trials for the National Cholesterol Education Program Adult Treatment Panel III guidelines. Circulation. 2004;110:227-39.

20. Banach M, Kourliouros A, Reinhart KM, Benussi S, Mikhailidis DP, Jahangiri M, et al. Postoperative atrial fibrillation-what do we really know? Curr Vasc Pharmacol. 2010;8:553-72.

21. Young-Xu Y, Jabbour S, Goldberg R, Blatt CM, Graboys T, Bilchik B, et al. Usefulness of statin drugs in protecting against atrial fibrillation in patients with coronary artery disease. Am J Cardiol. 2003;92:1379-83.

22. Kourliouros A, Roberts N, Jahangiri M. Statins with equivalent lipid-lowering capacity exhibit differential effects on atrial fibrillation after cardiac surgery. J Thorac Cardiovasc Surg. 2008;136:1100-1; author reply 1. 